\title{
Difference Grey Predictor Compensated Moving Sliding Mode Controller of a Digital Signal Processing-Based Solar Powered Communication Systems
}

\author{
En-Chih Chang*, Hsiao-Sheng Chen, Rong-Ching Wu, Guan-Yu Chen \\ Department of Electrical Engineering, I-Shou University, No. 1, Sec. 1, Syuecheng Rd., Dashu District, \\ Kaohsiung City 84001, Taiwan, R.O.C. \\ * Corresponding author. Tel: +886-7-6577711 Ext. 6642; email: enchihchang@isu.edu.tw \\ Manuscript submitted July 21, 2015; accepted October 15, 2015. \\ doi: 10.17706/ijcee.2015.7.5.350-356
}

\begin{abstract}
In this paper, a difference grey predictor (DGP) compensated moving sliding mode controller (MSMC) for solar powered communication systems. The proposed methodology combines the merits of DGP and MSMC. The MSMC can shorten the reaching phase and ensure the sliding mode occurrence from an arbitrary initial state. However, as the loading is a severe nonlinear condition, the MSMC will undergo chatter and steady-state error problems, thus deteriorating the performance of solar powered communication systems. The DGP is thus used to remove the chatter while the system uncertainty bounds are overestimated and to reduce the steady-state error while the system uncertainty bounds are underestimated. With the proposed methodology, the robustness of the communication system can be enhanced expectably, and a high-quality solar power sinusoidal output with low voltage harmonics and fast dynamic response can be obtained even under nonlinear loading. The theoretic analysis, design process, and digital signal processing (DSP)-based experimental implementation for solar powered communication systems are presented to verify the efficacy of the proposed methodology.
\end{abstract}

Key words: Difference grey predictor (DGP), moving sliding mode controller (MSMC), solar powered communication systems, voltage harmonics, digital signal processing (DSP).

\section{Introduction}

Communication systems have been widely used in the daily life, such as cellular phones, satellite communications, and radios. Once natural catastrophes such as blizzards, windstorms, and deluges occur, the power systems may be destroyed and then the communication system cannot be used. For continued communication operations, solar power is available and has the ability to meet critical power needs. In solar energy systems, the overall performance is dependent upon the static inverter-filter arrangement, which can be used to convert a DC voltage to a sinusoidal AC output. The requirements for a high performance solar power must provide high-quality AC output voltage of low total harmonic distortion (THD), zero steady-state errors and fast dynamic response, and these can be obtained by employing feedback control techniques. The linear proportional Integral (PI) controller is often used. However, the classic PI controlled system can not assure fast and stable output-voltage response [1], [2]. Nonlinear control theory has to be employed and the use of sliding mode control (SMC) is a proper choice because the SMC offers the insensitivity to system uncertainties [3], [4]. In the design of the classic SMC with fixed sliding surface, there is a reaching phase in which the trajectory starting from a given initial state off the sliding surface tends towards the sliding surface. 
To overcome such a problem, the MSMC is used to pass arbitrary initial conditions and afterward moves towards a designated sliding surface by the concept of rotating or shifting. [5], [6]. Using MSMC with the moving sliding surface, the system will be more insensitive to load disturbances because of shortening the reaching phase. However if a highly nonlinear loading is applied, the MSMC controlled system may occur chatter and steady-state errors, thus resulting in serious solar power output voltage harmonics, even deteriorates the performance of solar powered communication systems. The grey predictor (GP) has been presented and displays many applications in a variety of fields. [7], [8]. This paper employs a mathematically simple and computationally efficient DGP to remove the chatter or steady-state errors when the system uncertainties bounds are overestimated or underestimated. By combining MSMC and DGP, the proposed methodology will yield a closed-loop solar powered communication system with low THD, fast dynamic response, chatter removal, and steady-state errors reduction under various loading. Experimental results are shown to testify the performance of the proposed methodology.

\section{System Description}

The structure of a static power supply, shown in Fig. 1 can be used in solar powered communication system. The $L C$ filter and $R$ can be regarded as a plant. The output voltage $v_{c}$ of the plant should be forced to track a sinusoidal reference waveform $v_{r}$ by applying the proposed methodology.

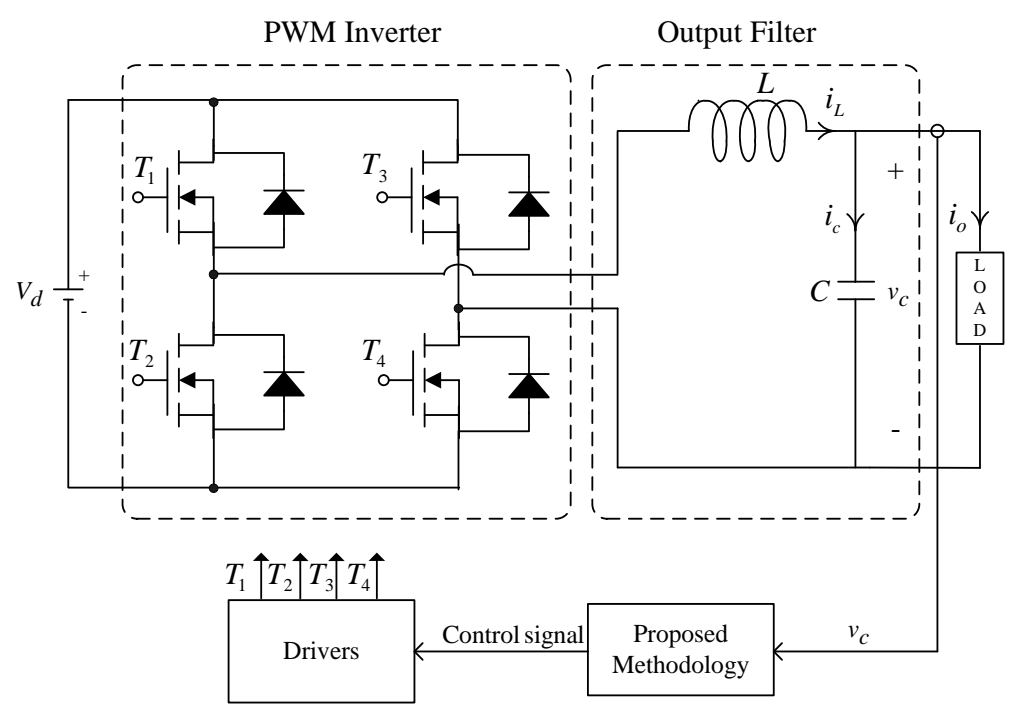

Fig. 1. Circuit diagram of solar powered communication system.

\section{Controller Design}

\subsection{Moving Sliding Mode Controller (MSMC)}

The plant can be written as

$$
\dot{x}_{p}=A_{p} x_{p}+B_{p} u_{p}+w
$$

where $x_{p}=\left[\begin{array}{ll}x_{p 1} & x_{p 2}\end{array}\right]^{T}=\left[\begin{array}{ll}v_{c} & \dot{v}_{c}\end{array}\right]^{T}, A_{p}=\left[\begin{array}{cc}0 & 1 \\ -\frac{1}{L C} & -\frac{1}{R C}\end{array}\right], B_{p}=\left[\begin{array}{c}0 \\ \frac{1}{L C}\end{array}\right], u_{p}$ is the control input of plant, and $w$ is the system uncertainty. 
The sinusoidal reference model can be expressed as

$$
\dot{x}_{m}=A_{m} x_{m}+B_{m} u_{m}
$$

where $x_{m}=\left[\begin{array}{ll}x_{m 1} & x_{m 2}\end{array}\right]^{T}=\left[\begin{array}{ll}v_{r} & \dot{v}_{r}\end{array}\right]^{T}, A_{m}=\left[\begin{array}{cc}0 & 1 \\ \omega_{0}^{2} & 0\end{array}\right], B_{m}=\left[\begin{array}{l}0 \\ 1\end{array}\right], \omega_{0}$ is the frequency of the sinusoidal reference waveform, and $u_{m}$ is the control input of the sinusoidal reference model.

The error state equations can be represented as

$$
\begin{gathered}
e=x_{m}-x_{p} \\
\dot{e}=A_{m} e+\left(A_{m}-A_{p}\right) x_{p}+B_{m} u_{m}-B_{p} u_{p}-w
\end{gathered}
$$

Then, construct the MSMC function as

$$
\sigma=c(t) e-\alpha(t)
$$

where $c(t)=A t+B, \alpha(t)=C t+D$, and $A, B, C, D$ are constants.

Differentiating (5), we have

$$
\dot{\sigma}=A e_{1}+c(t) \dot{e}_{2}+\dot{e}_{2}-C
$$

The control input is set as

$$
u_{p}=u_{e}+u_{s}
$$

where the $u_{e}$ is the equivalent control, which guarantees the realization of desired sliding mode when system uncertainty is equal to zero, i.e., $\left.\dot{\sigma}\right|_{u_{p}=u_{e}}=0$; the $u_{s}$ is the nonlinear control part, which is used to remove the system uncertainty so that the realization of sliding mode can be guaranteed.

The function $u_{s}$ is created as

$$
u_{s}=K e+K_{n}
$$

\subsection{Difference Grey Predictor (DGP)}

The GM(2,1) is used to build a grey prediction model and the grey modeling steps are described below. Step 1: Collect the original sample data sequence as

$$
x^{(0)}=\left\{x^{(0)}(k), \quad k=1,2, \ldots, n\right\}
$$

Step 2: Mapping generating operation (MGO): Negative data is mapped to the relative positive data. 


$$
x_{\text {new }}^{(0)}=\left\{x^{(0)}(k)+\text { bias }, \quad k=1,2, \ldots, n\right\}
$$

where bias is constant.

Step 3: Take Accumulated generating operation (AGO) for $x_{\text {new }}^{(0)}$ as

$$
x_{\text {new }}^{(1)}(k)=\sum_{i=1}^{k} x_{\text {new }}^{(0)}(i), \quad k=1,2, \ldots, n
$$

Step 4: Construct GM(2,1) model (second-order difference equation) as

$$
x_{\text {new }}^{(1)}(k+2)+U x_{\text {new }}^{(1)}(k+1)+W x_{\text {new }}^{(1)}(k)=0
$$

where $U$ and $W$ are the coefficients of the $\operatorname{GM}(2,1)$ model and they must be estimated. Thus, letting $k=1,2, \ldots, n-2$ and then the (13) becomes

$$
\underbrace{\left[\begin{array}{cc}
-x_{\text {new }}^{(1)}(2) & -x_{\text {new }}^{(1)}(1) \\
-x_{\text {new }}^{(1)}(3) & -x_{\text {new }}^{(1)}(2) \\
\vdots & \vdots \\
-x_{\text {new }}^{(1)}(n-1) & -x_{\text {new }}^{(1)}(n-2)
\end{array}\right]}_{B} \underbrace{\left[\begin{array}{c}
U \\
W
\end{array}\right]}_{\Omega}=\underbrace{\left[\begin{array}{c}
x_{\text {new }}^{(1)}(3) \\
x_{\text {new }}^{(1)}(4) \\
\vdots \\
x_{\text {new }}^{(1)}(n)
\end{array}\right]}_{Y}
$$

Finally, by the least square estimation method, the $U$ and $W$ can be solved as $\Omega=[U, W]^{T}=\left(B^{T} B\right)^{-1} B^{T} Y$. To find the solution of $\Omega$, we define $x_{n e w}^{(1)}(k)=\rho^{k}, x_{n e w}^{(1)}(k+1)=\rho^{k+1}$ and $x_{\text {new }}^{(1)}(k+2)=\rho^{k+2}$, and then the following equation yields $\rho^{k+2}+U \rho^{k+1}+W \rho^{k}=\rho^{k}\left(\rho^{2}+U \rho+W\right)=0$.

Step 5: Take Inverse accumulated generating operation (IAGO) as

$$
\hat{x}_{\text {new }}^{(0)}(k)=\hat{x}_{\text {new }}^{(1)}(k)-\hat{x}_{\text {new }}^{(1)}(k-1)
$$

Step 6: Inverse mapping generating operation (IMGO): By applying IMGO for $\hat{x}_{n e w}^{(0)}$, the predicted value of the original data sequence $\hat{x}^{(0)}$ yields

$$
\hat{x}^{(0)}(k+1)=\hat{x}_{\text {new }}^{(1)}(k+1)-\hat{x}_{\text {new }}^{(1)}(k)-\text { bias }
$$

Therefore, the difference grey prediction control can be obtained to remove the chatter or to reduce the steady-state error as follows: $u_{d g}(k)=\left\{\begin{array}{cl}0 & ,|\hat{\sigma}(k)|<\phi \\ K \hat{\sigma}(k) \operatorname{sgn}(\sigma(k) \hat{\sigma}(k)) & , \quad|\hat{\sigma}(k)| \geq \phi\end{array}\right.$, where $K$ is constant, $\hat{\sigma}(k)$ denotes the predicted value of $\sigma(k)$ and $\phi$ represents the system boundary.

\section{Experimental Results}


The system parameters are given as follows: DC-bus Voltage $V_{d}=200 \mathrm{~V}$; Output Voltage $v_{c}=110 \mathrm{~V}$; Output Frequency $f=60 \mathrm{~Hz}$; Filter Inductor $L=1.5 \mathrm{mH}$; Filter Capacitor $C=15 \mu \mathrm{F}$; Switching Frequency $f_{s}=15 \mathrm{kHz}$; Rated Load $R=12 \Omega$. Fig. 2(a) show the output voltage and the load current with the proposed methodology under step change in load from open circuit to $R=12 \Omega$. Clearly, a fast recovery of the steady-state response is obtained. However, the waveform with the classic SMC, shown in Fig. 2(b) has poor voltage dip compensation at the firing angle. In addition, the majority of sensitive loads are rectifier loads. Therefore, the proposed methodology must correctly regulate output voltage with minimum distortion. Fig. 2(c) shows both the output voltage and the voltage current with the proposed methodology when a full-wave rectifier followed by a $100 \mu \mathrm{F}$ capacitor in parallel with a $50 \Omega$ resistor is applied; the \%THD is close to $1.17 \%$, which displays a good system performance. Oppositely, Fig. 2(d) with the classic SMC under the same test condition exhibits a high voltage $\%$ THD of $7.42 \%$.

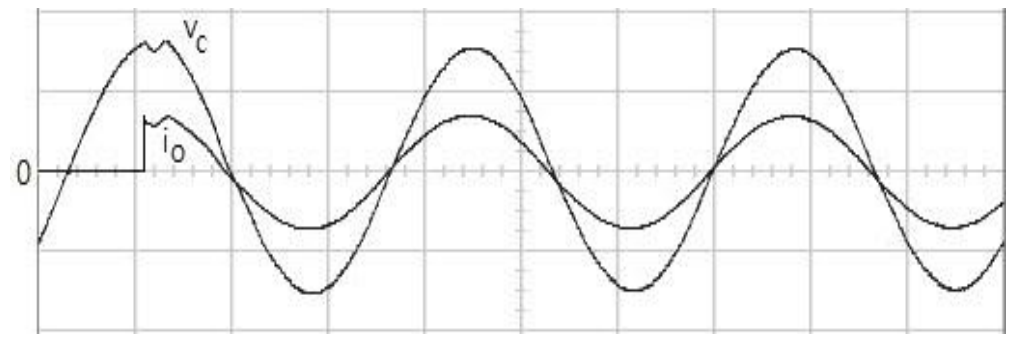

(a) $(100 \mathrm{~V} / \mathrm{div} ; 20 \mathrm{~A} / \mathrm{div} ; 5 \mathrm{~ms} / \mathrm{div})$

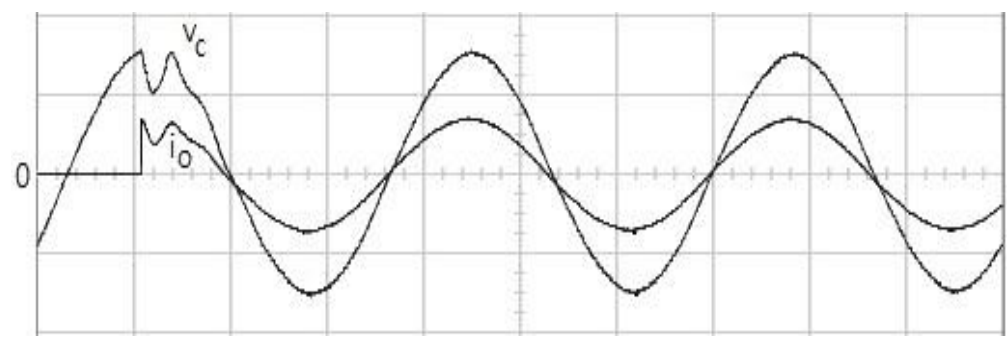

(b) $(100 \mathrm{~V} / \mathrm{div} ; 20 \mathrm{~A} / \mathrm{div} ; 5 \mathrm{~ms} / \mathrm{div})$

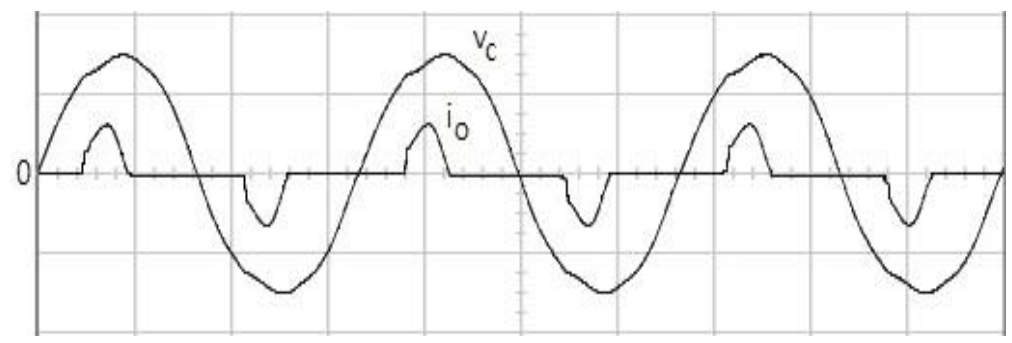

(c) $(100 \mathrm{~V} / \mathrm{div} ; 25 \mathrm{~A} / \mathrm{div} ; 5 \mathrm{~ms} / \mathrm{div})$

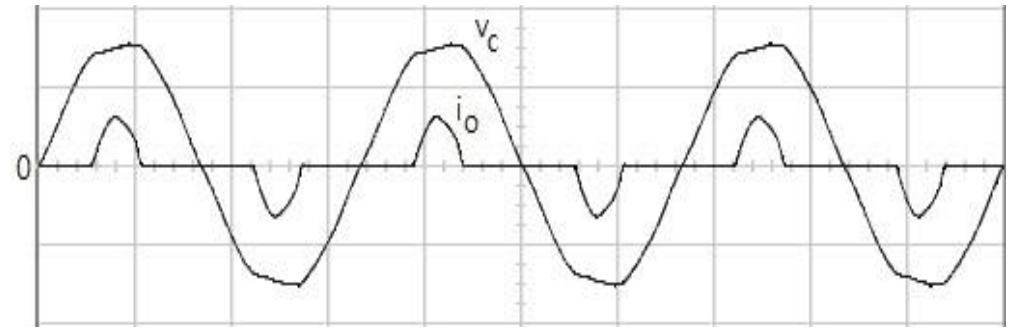

(d) (100V/div; 25A/div; 5ms/div)

Fig. 2. Output waveforms under. (a) Step change in load with the proposed methodology. (b) Step change in load with the classic SMC. (c) Rectifier load with proposed methodology. (d) Rectifier load with the classic SMC. 


\section{Conclusion}

A DGP compensated MSMC is proposed to improve the tracking behaviors of the solar powered communication systems. Compared with classic SMC, the MSMC shortens the reaching phase and increases the insensitivity to system uncertainties. For achieving high tracking accuracy, the DGP is used to remove the chatter or reduce the steady-state error, which is produced by MSMC. Experimental results indicate that low harmonic distortion and fast transient response are obtained in the proposed controlled solar powered communication system under both steady-state loading and transient loading.

\section{Acknowledgment}

This work was supported by the Ministry of Science and Technology of Taiwan, R.O.C., under contract number MOST104-2221-E-214-011.

\section{References}

[1] Rasoanarivo, I., \& Sargos, F. (2013). Multi-objective analysis for designing and controlling micro-grids under multi-control with PID, MHCC and FOPID controllers. Proceedings of the IEEE Int. Conf. Industry Applications Society Annual Meeting (pp. 1-8).

[2] Rebeiro, R. S., \& Uddin, M. N. (2012). Performance analysis of an FLC-based online adaptation of both hysteresis and PI controllers for IPMSM drive. IEEE Trans. on Industry Applications, 44(1), 12-19.

[3] Itkis, U. (1976). Control Systems of Variable Structure. New York: Wiley.

[4] Aghatehrani, R., \& Kavasseri, R. (2013). Sensitivity-analysis-based sliding mode control for voltage regulation in micro-grids. IEEE Trans. on Sustainable Energy, 4(1), 50-57.

[5] Yorgancıŏglu, F., \& Kömürcügil, H. (2008). Single-input fuzzy-like moving sliding surface approach to the sliding mode control. Electrical Engineering, 90(3), 199-207.

[6] Li, L., Zhang, Q. Z., \& Rasol, N. (2011). Time-varying sliding mode adaptive control for rotary drilling system. Journal of Computers, 6(3), 564-570.

[7] Liu, S., \& Lin, Y. (2010). Advances in Grey Systems Research. Berlin: Springer-Verlag.

[8] Wang, D., Huang, X., Wang, M. D., \& Liu, T. B. (2013). Application of gray GM(2,1) model to prediction of landslide deformation. Hydrogeology and Engineering Geology, 40(3), 121-125.

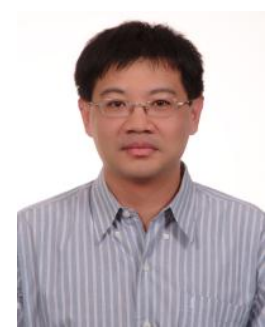

En-Chih Chang was born in Kaohsiung, Taiwan, in 1975. He received the B.S. degree from Feng-Chia University, Taichung, Taiwan, R.O.C., in 1999, the M.S. degree from National Taiwan Ocean University, Keelung, Taiwan, R.O.C., in 2001, and the Ph.D. degree from National Cheng Kung University, Tainan, Taiwan, R.O.C., in 2008, all in electrical engineering. He joined the Department of Electrical Engineering, I-Shou University in 2009 as an assistant professor. His research interests include sliding mode control, intelligent control, grey theory, and their applications in power electronics systems.

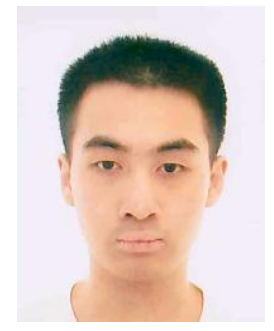

Hsiao-Sheng Chen was born in Chiayi City, Taiwan, in 1995. He is working toward the B.S. degree in electrical engineering at I-Shou University, Dashu District, Kaohsiung City, Taiwan, R.O.C. His research interests include intelligent control, intelligent robotics, and the embedded systems. 
Rong-Ching Wu graduated from National Taiwan Institute of Technology, Taipei, Taiwan, in 1990. He received the M.Sc. degree in 1994 and the Ph.D. degree in 2001 from National Sun Yat-Sen University, Kaohsiung, Taiwan, R.O.C. From 1991 to 2001, he was an electrical engineer in Taiwan Power Company, Kaohsiung, Taiwan, and was involved with design, construction, and operation of power systems. Currently, he is an assistant professor at I-Shou University, Kaohsiung, Taiwan, R.O.C.

Guan-Yu Chen was born in Kaohsiung, Taiwan, in 1994. He is working toward the B.S. degree in electrical engineering at I-Shou University, Dashu District, Kaohsiung City, Taiwan, R.O.C. His research interests include intelligent control, and the field of power electronics. 